05

\title{
О зависимости энтальпии прямого мартенситного превращения в никелиде титана от напряжения
}

\author{
(c) C.А. Егоров, А.Е. Волков
}

Санкт-Петербургский государственный университет, 198905 Санкт-Петербург, Россия

e-mail: truhtin@mail.ru

(Поступило в Редакцию 12 апреля 2016 г. В окончательной редакции 13 июля 2016 г.)

Создана оригинальная методика дифференциального термического анализа для изучения теплофизических свойств образцов, нагруженных касательным напряжением. В серии экспериментов по исследованию прямого мартенситного превращения $B 2 \rightarrow B 19^{\prime}$ в никелиде титана при охлаждении под постоянным напряжением обнаружено, что энтальпия превращения линейно убывает с ростом напряжения, и при напряжении $100 \mathrm{MPa}$ она на $30 \%$ меньше, чем у образца в свободном состоянии.

DOI: 10.21883/JTF.2017.02.44126.1855

\section{Введение}

В последнее время возник интерес к явлению, которое обнаружено в калориметрических опытах со сплавами, испытывающими мартенситные превращения. Это явление заключается в уменьшении абсолютного значения энтальпии превращения $q$ (для прямого превращения $q<0)$ под действием напряжений $\sigma$.

Энтальпия превращения равна скачку энтальпии в результате преобразования аустенита в мартенсит $q=[W]=W^{\mathrm{M}}-W^{\mathrm{A}}$, где $W^{\mathrm{M}}, W^{\mathrm{A}}-$ энтальпии (на единицу объема) мартенситной и аустенитной фаз. Из первого начала термодинамики и определения энтальпии следует

$$
d W=\delta Q-\varepsilon: d \sigma,
$$

где $\delta Q-$ подведенное тепло, $\sigma$ и $\varepsilon-$ тензоры напряжений и малой деформации напряжения, а знак ,„“ обозначает свертку тензоров. Рассчитаем по отдельности энтальпии мартенсита и аустенита.

В отсутствии превращения мартенсит (аустенит) испытывает обратимые процессы теплообмена, упругой деформации и теплового расширения. При этом $\delta Q=T d S$, где $T$ - температура, $S$ - энтропия, следовательно,

$$
d W=T d S-\varepsilon: d \sigma .
$$

Предположим, что тензоры коэффициентов теплового расширения мартенсита и аустенита $\alpha^{\mathrm{M}}$ и $\alpha^{\mathrm{A}}$, упругих податливостей $D^{\mathrm{M}}$ и $D^{\mathrm{A}}$, а также удельные теплоемкости при постоянном напряжении $c_{\sigma}^{\mathrm{M}}$ и $c_{\sigma}^{\mathrm{A}}$ не зависят от температуры и напряжения. Связь между деформацией и напряжением для материала в аустенитном (А) и мартенситном (М) состояниях зададим обычным законом Дюгамеля-Неймана:

$$
\begin{gathered}
\varepsilon^{\mathrm{A}}(T, \sigma)=\varepsilon_{1}^{\mathrm{A}}+\left(T-T_{1}\right) \alpha^{\mathrm{A}}+D^{\mathrm{A}}:\left(\sigma-\sigma_{1}\right), \\
\varepsilon^{\mathrm{M}}(T, \sigma)=\varepsilon_{1}^{\mathrm{M}}\left(\sigma_{1}\right)+\left(T-T_{1}\right) \alpha^{\mathrm{M}}+D^{\mathrm{M}}:\left(\sigma-\sigma_{1}\right),
\end{gathered}
$$

где $T_{1}, \sigma_{1}$ - температура и напряжение, при которых образовался мартенсит.
В формуле (2) за нуль примем деформацию аустенита при $T=T_{1}, \sigma=\sigma_{1}$, т.е. положим $\varepsilon_{1}^{\mathrm{A}}=0$, тогда в формуле (3) $\varepsilon_{1}^{\mathrm{M}}\left(\sigma_{1}\right)$ есть деформация мартенсита по отношению к аустениту (фазовая деформация), равная скачку деформации при превращении. Отметим, что скачок макроскопической деформации зависит от напряжения, поэтому формула (3) дает деформацию мартенсита как функцию $T$ и $\sigma$ только при условии, что он образовался при $T=T_{1}, \sigma=\sigma_{1}$. Дифференциал энтропии как функции $T$ и $\sigma$ выражается формулой

$$
\begin{aligned}
d S^{a} & =\left(\frac{\partial S^{a}}{\partial T}\right)_{\sigma} d T+\left(\frac{\partial S^{a}}{\partial \sigma}\right)_{T}: d \sigma \\
& =\left(\frac{c_{\sigma}}{T}\right) d T+\left(\frac{\partial \varepsilon^{a}}{\partial T}\right)_{\sigma}: d \sigma \\
& =\left(\frac{c_{\sigma}^{a}}{T}\right) d T+\alpha^{a}: d \sigma, \quad a=A, M
\end{aligned}
$$

где верхний индекс $a=\mathrm{A}$ соответствует аустениту, $a=\mathrm{M}$ - мартенситу. В формуле (4) применена принятая в термодинамике форма записи, в которой нижний индекс у частной производной обозначает величину, постоянную при дифференцировании, и учтено соотношение между термодинамическими коэффициентами $(\partial S / \partial \sigma)_{T}=(\partial \varepsilon / \partial T)_{\sigma}$, вытекающее из условия полного дифференциала потенциала Гиббса $d G=-S d T-\varepsilon: d \sigma$. Подставляя (2)-(4) в (1), и интегрируя от $T=T_{1}$, $\sigma-\sigma_{1}=0$ до $T, \sigma$, найдем энтальпии фаз

$$
\begin{gathered}
W^{a}(T, \sigma)=W^{a}\left(T_{1}, \sigma_{1}\right)+c_{\sigma}^{a}\left(T-T_{1}\right)-\varepsilon_{1}^{a}:\left(\sigma-\sigma_{1}\right) \\
+T_{1} \alpha^{a}:\left(\sigma-\sigma_{1}\right)-(1 / 2)\left(\sigma-\sigma_{1}\right): D^{a}:\left(\sigma-\sigma_{1}\right), \\
a=\mathrm{A}, \mathrm{M} .
\end{gathered}
$$

Из (5) найдем разность энтальпий аустенита и мартенсита

$$
\begin{aligned}
& {[W(T, \sigma)]=\left[W\left(T_{1}, \sigma_{1}\right)\right]+\left[c_{\sigma}\right]\left(T-T_{1}\right)-\left[\varepsilon_{1}\right]:\left(\sigma-\sigma_{1}\right)} \\
& +T_{1}[\alpha]:\left(\sigma-\sigma_{1}\right)-(1 / 2)\left(\sigma-\sigma_{1}\right):[D]:\left(\sigma-\sigma_{1}\right),
\end{aligned}
$$

где квадратные скобки обозначают разницу значений соответствующей величины для мартенсита и аустенита, 
иными словами - ее скачок при переходе от аустенита к мартенситу. Подставляя в (6) $T=T_{1}, \sigma=\sigma_{0}=0$ и пренебрегая слагаемыми, содержащими скачки $\left[c_{\sigma}\right],[\alpha]$, $[D]$, найдем

$$
\left[W\left(T_{1}, \sigma_{1}\right)\right]=\left[W\left(T_{1}, \sigma_{0}\right)\right]-\left[\varepsilon_{1}\right]: \sigma_{1}
$$

Или в обозначениях $q_{1}=\left[W\left(T_{1}, \sigma_{1}\right)\right], q_{0}=\left[W\left(T_{1}, \sigma_{0}\right)\right]$,

$$
q_{1}=q_{0}-\left[\varepsilon_{1}\right]: \sigma_{1} \text {. }
$$

Поскольку напряжение вызывает фазовую деформацию, такую, что $\sigma:\left[\varepsilon_{1}\right]>0$, и учитывая, что $q<0, q_{0}<0$, имеем $|q|>\left|q_{0}\right|$, т. е. под нагрузкой абсолютная величина энтальпии превращения должна увеличиваться.

Этот вывод соответствует теоретическим $[1,2]$ и экспериментальным [3] исследованиям, выполненным на образцах из монокристалла $\mathrm{CuAlNi}$, но противоречит экспериментальным наблюдениям, полученным для поликристалла TiNi [4] и для монокристалла CuAlNi [5]. Важно, что авторы работ $[1,2]$ допускают, что фиксируемое калориметром тепловыделение при превращении под напряжением $\sigma \neq 0$ может быть меньше, чем при $\sigma=0$, поскольку внутренняя энергия мартенситной фазы включает в себя энергию границ ориентационных доменов мартенсита, число которых тем меньше, чем больше механическое напряжение. Данное рассуждение показывает, что напряжение может в принципе приводить к уменьшению абсолютной величины энтальпии превращения. Таким образом, напряжение влияет на энтальпию превращения неоднозначно, и теоретический анализ, кроме использования соотношения (8), должен включать оценку величины удельной поверхностной энергии междоменных границ, анализ зависимости их суммарной площади от напряжения и оценку энергии внутренних напряжений, обусловленных несовместностью фазовых превращений.

Другое объяснение было дано в [4] на основе рассуждения о том, что скачок энтропии при превращении, начиная с некоторого критического напряжения, может уменьшаться вследствие вызванного им изменения симметрии кристаллов. Это предположение своим следствием может иметь нелинейный, и даже скачкообразный характер зависимости внутренней теплоты фазового полиморфного перехода от напряжений. В работе [3] было эксперименртально показано, что при растяжении монокристалла из $\mathrm{CuAlNi}$ эта зависимость линейная, но до сих пор остается неясной ситуация для других случаев. Все это указывает на необходимость дальнейших исследований по данной тематике.

Важно отметить, что во всех известных работах по данному вопросу использовали калориметрические приборы (DSC-метод), и нагрузку в образцах создавали не путем непосредственного нагружения, а посредством их защемления. Напряжения возникали как проявление эффекта памяти формы в режиме стесненной деформации внутри калориметрической ячейки: при нагреве через интервал обратного мартенситного превращения в предварительно деформированном образце вследствие его защемления имеет место генерация реактивных напряжений, а при охлаждении через интервал прямых превращений происходит их релаксация. При такой схеме проведения опыта напряжения во время термоциклирования не постоянны. Кроме того, их величину нельзя непосредственно измерить, а можно лишь приблизительно оценить расчетным путем. Наконец, действуя таким образом, невозможно осуществить эксперименты с одним и тем же материалом, в которых в рамках одной и той же схемы напряженного состояния реализовывались бы ситуации с разными по величине напряжениями. Иными словами, методика, основанная на DSC, не позволяет экспериментально найти зависимость энтальпии перехода от действующего напряжения. В работе [6] представлен оригинальный прибор DSC, позволяющий изучать величину энтальпии превращения под напряжением, но только при одноосном растяжении. Для других схем напряженного состояния соответствующих приборов до сих пор не было.

Таким образом, актуальность проблемы влияния напряжений на энтальпию мартенситных превращений объединяется с актуальностью проблемы создания соответствующей экспериментальной методики для случая, отличающегося от одноосного растяжения. Поэтому в рамках настоящей работы была разработана методика изучения материала при кручении, основанная на принципах дифференциального термического анализа (ДТА), изготовлена соответствующая конструкция и выполнены эксперименты, направленные на изучение влияния напряжений на энтальпию прямого мартенситного превращения в образцах никелида титана.

\section{Методика проведения исследований}

Для проведения опытов была разработана и реализована методика, представляющая собой усовершенствованный вариант ДТА. Классическая схема ДТА это измерение разности температур между образцом и эталоном (в первом есть фазовый переход, а во втором его нет) в зависимости от температуры образца. При таком способе исследования можно организовать разнообразные виды воздействия (в том числе механического) на образец, хорошо определять температуру начала и приблизительно температуру окончания фазовой реакции, но невозможно определить как величину энтальпии перехода.

В разработанной методике сохраняются все положительные черты классического ДТА и прибавляется возможность оценивать величину тепловой реакции и определять ее изменение в зависимости от приложенного механического напряжения в режиме кручения. Схема установки для термомеханических испытаний дана на рис. 1. Она представляет собой соосную конструкцию, в которой трубчатый образец 2 и такой же формы и размеров эталон 4 вставлены в валы 1,3 и 5 из пластика, 


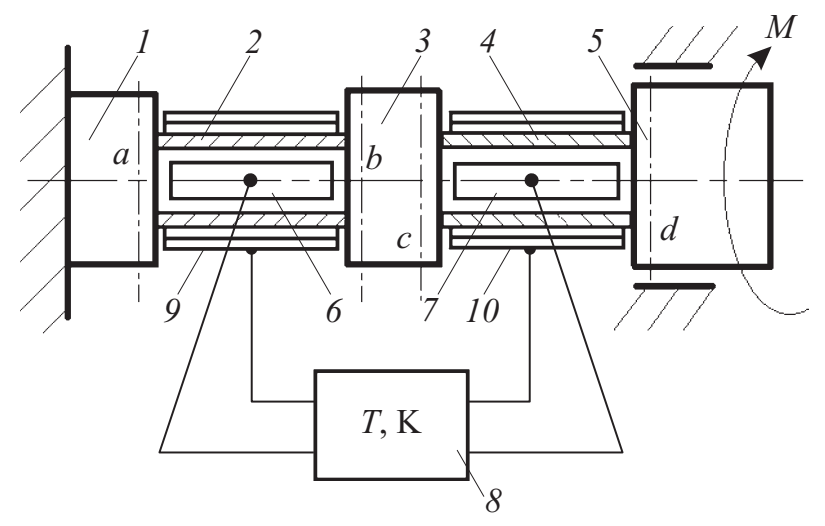

Рис. 1. Схема установки для проведения термомеханических испытаний: 1,3,5 - валы из термопрочного пластика, 2 - образец, 4 - эталон, 6, 7 - цилиндрические медные вставки, 9, 10 - трубчатые медные вставки, 8 - четырехканальный температурометр. Линиями $a, b, c, d$ обозначены стальные крепежные стержни.

сохраняющего свои высокие прочностные свойства до температуры $600 \mathrm{~K}$. Пластик выбран для уменьшения коэффициента теплообмена и соответственно получения более однородного поля температур в образце и эталоне по сравнению с использованием любого конструкционного металла. Отметим, что трение этого пластика о металл основания (конструкционная сталь) весьма мало, в результате чего реактивные напряжения в образце во время проведения опытов были пренебрежимо малыми (не превышали 1\% от действующих напряжений). Эталон изготовлен из нержавеющей стали без фазовых переходов в диапазоне температур 250-700 К. Крепление образца 2 и эталона 4 к валам 1,3,5 осуществляется посредством стержней из нержавеющей стали, пронзающих насквозь соединяемые детали в поперечном направлении (условно обозначено вертикальными штрихпунктирными линиями $a-d$ ). Через вал 5 на всю конструкцию передается крутящий момент, а по углу его поворота определяется деформация кручения. Во время нагревания и охлаждения измеряются температуры медных вставок: внутренних цилиндрических вставок 6, 7 и внешних вставок 9, 10 (размеры в каждой этой паре одинаковые). Каждая из вставок 9 и 10 состоит из двух одинаковых половинок, которые при совместном наложении на объект создают внешнюю оболочку в виде трубки. Надежный термический контакт образца и эталона с соответствующими вставками обеспечивается с помощью теплопроводной пасты с окисью бериллия. Отметим, что общая масса этой пасты на два порядка меньше общей массы используемых медных вставок, так что ее теплоемкость не влияет на результаты исследования. Температура измеряется хромель-копелевыми термопарами в местах, обозначенных на схеме большими черными точками, с помощью четырехканального электронного температурометра 8 . Таким образом, как на стороне образца, так и на стороне эталона с заданным интервалом времени $\Delta t$ регистрируются значения температур внутри (в центре) внутренних вставок и снаружи внешних вставок - всего четыре одновременных измерения. Образец и эталон вместе с наружными медными вставками теплоизолированы от окружающей среды, каждый отдельно, но одинаковым способом. Вся конструкция закрыта кожухом и установлена на плоском электронагревательном элементе (на схеме не показаны). В начале опыта на нагревателе устанавливается фиксированное значение электрического напряжения $E$, которое не меняется до конца нагревания. В начале нагрева в конструкции установки возникает сильно неравновесное термическое состояние - как из-за излучения, так и вследствие возникновения конвекционных потоков воздуха. Поэтому величину $E$ подбирали так, чтобы к началу обратной реакции все термические процессы шли равномерно, и, следовательно, характеристические температуры фазового превращения надежно фиксировались. Тоже самое относится и к этапу охлаждения: одномоментное уменьшение величины $E$ выбирали таким, чтобы охлаждение перед началом фазового перехода представляло собой равномерный и установившийся процесс. В описанных ниже опытах охлаждение до комнатной температуры 290-295 К происходило самопроизвольно вследствие полного выключения нагревателя (без стимулирования этого процесса).

В результате измерений температур медных вставок с удовлетворительной точностью определяли температуры образца и эталона. Действительно, поскольку теплопроводность медных вставок в интервале рабочих температур $(300-500 \mathrm{~K})$ велика, а их размеры и массы малы (в данных опытах их масса была 4-6g), с высокой степенью точности можно сказать, что температура образца (эталона) есть среднее значение между температурами соответствующих внутренней и внешней вставок. Это тем более оправдано, что, как показали опыты, даже во время фазового перехода разница этих температур не превышает $1.5 \mathrm{~K}$. Прямые тестовые проверки показали, что погрешность такого опосредованного способа определения температуры не более $0.2 \mathrm{~K}$.

Для расчета скорости притока (оттока) тепла к образцу предполагали, что теплообмен происходит через захваты (боковые поверхности были теплоизолированы асбестом) и подчиняется ньютоновскому закону

$$
Q_{k}=H\left(T_{g}-T_{\mathrm{ON} k}\right), \quad k=1,2,
$$

где индекс $k=1$ - соответствует образцу, $k=2-$ эталону, $Q_{k}$ - скорость притока тепла, $T_{\mathrm{ON} k}-$ температура внешней медной вставки, $T_{g}$ - температура захватов, $H$ - коэффициент теплообмена, одинаковый для образца и эталона.

Для экспериментального определения коэффициента $H$ отдельно выполнили опыт, в котором осуществляли нагрев эталона с измерением зависимостей $T_{g}(t)$ и $T_{\mathrm{ON} 2}(t)$. Для такого опыта баланс тепла записывается 
в виде

$$
\begin{aligned}
H\left(T_{g}-T_{\mathrm{ON} 2}\right)= & C_{\mathrm{Cu}}\left(T_{\mathrm{ON} 2}\right) m_{\mathrm{ON}} \dot{T}_{\mathrm{ON} 2} \\
& +C_{\mathrm{Cu}}\left(T_{\mathrm{IN} 2}\right) m_{\mathrm{IN}} \dot{T}_{\mathrm{IN} 2}+C_{2}\left(T_{2}\right) m_{2} \dot{T}_{2},
\end{aligned}
$$

где $C_{\mathrm{Cu}}(T), C_{2}(T)$ - удельные теплоемкости меди и эталона при температуре $T, m_{\mathrm{ON}}$ и $m_{\mathrm{IN}}-$ массы внешней и внутренней медных вставок, $T_{2}-$ температура эталона, для которой принимали оценку $T_{2}=\left(T_{\mathrm{ON} 2}+T_{\mathrm{IN} 2}\right) / 2$. Точка над буквой обозначает производную по времени. Кроме того, приближенно считали, что $C_{\mathrm{Cu}}\left(T_{\mathrm{IN} 2}\right)=C_{\mathrm{Cu}}\left(T_{\mathrm{ON} 2}\right)$ ввиду малой разницы между температурами $T_{\mathrm{IN} 2}$ и $T_{\mathrm{ON} 2}$.

После тестовых опытов с помощью уравнения (10) определили среднее значение коэффициента теплообмена $H=0.04 \mathrm{~W} / \mathrm{K}$.

Записывая для образца уравнение, аналогичное уравнению (10), и вычитая одно из другого, после очевидных преобразований найдем

$$
\begin{aligned}
C_{1}\left(T_{1}\right)= & \frac{1}{m_{1}}\left[C_{2}\left(T_{2}\right) m_{2} \frac{d T_{2}}{d T_{1}}-C_{\mathrm{Cu}}\left(T_{\mathrm{ON} 1}\right)\right. \\
& \left.\times \frac{d\left(m_{\mathrm{ON}} \Delta T_{\mathrm{ON}}+m_{\mathrm{IN}} \Delta T_{\mathrm{IN}}\right.}{d T_{1}}-\frac{H \Delta T_{\mathrm{ON}}}{\dot{T}_{1}}\right],
\end{aligned}
$$

где $\Delta T_{\mathrm{ON}}=T_{\mathrm{ON} 1}-T_{\mathrm{ON} 2}, \Delta T_{\mathrm{IN}}=T_{\mathrm{IN} 1}-T_{\mathrm{IN} 2}$.

Очевидно, что существующие и неустранимые в рамках данного подхода теплопотери не позволяют говорить о том, что он может составить конкуренцию классическим калориметрическим приборам и даст возможность точно определить величину $C_{1}(T)$, однако он позволяет изучать теплофизические свойства тел с памятью формы при нагревании и охлаждении с одновременным измерением деформации кручения под контролируемой нагрузкой.

\section{Экспериментальные результаты}

В качестве объекта исследований выбрали сплав $\mathrm{Ti}_{51.5} \mathrm{Ni}_{48.5}$ (at.\%), из которого изготовили трубчатый образец массой $3.12 \mathrm{~g}$, внешним диаметром $6 \mathrm{~mm}$, внутренним диаметром $5 \mathrm{~mm}$, длиной рабочей части $23 \mathrm{~mm}$ и двумя головками длиной по $10 \mathrm{~mm}$ с внешним диаметром $8 \mathrm{~mm}$. Масса $m$ образца была $3.12 \mathrm{~g}$. После закалки (выдержка при температуре $873 \mathrm{~K}$ в течение $1 \mathrm{~h}$, охлаждение в воде) пластинку материала массой $10 \mathrm{mg}$ протестировали на дифференциальном сканирующем калориметре DSC $822^{\mathrm{e}}$ METTLER TOLEDO с разрешением $0.04 \mu \mathrm{W}$. Изменение температуры осуществляли с темпом $5 \mathrm{grad} / \mathrm{min}$ в диапазоне температур 293-492 K. Оказалось, что при нагреве, начиная с температуры $A_{S}=378$ и кончая температурой $A_{F}=411 \mathrm{~K}$, наблюдается один пик теплопоглощения $q^{H}=31.8 \mathrm{~J} / \mathrm{g}$, при охлаждении в интервале температур от $M_{S}=340 \mathrm{~K}$ до $M_{F}=366 \mathrm{~K}$ - один пик тепловыделения $q^{C}=29.3 \mathrm{~J} / \mathrm{g}$. С учетом известных данных об изучаемом сплаве [7]
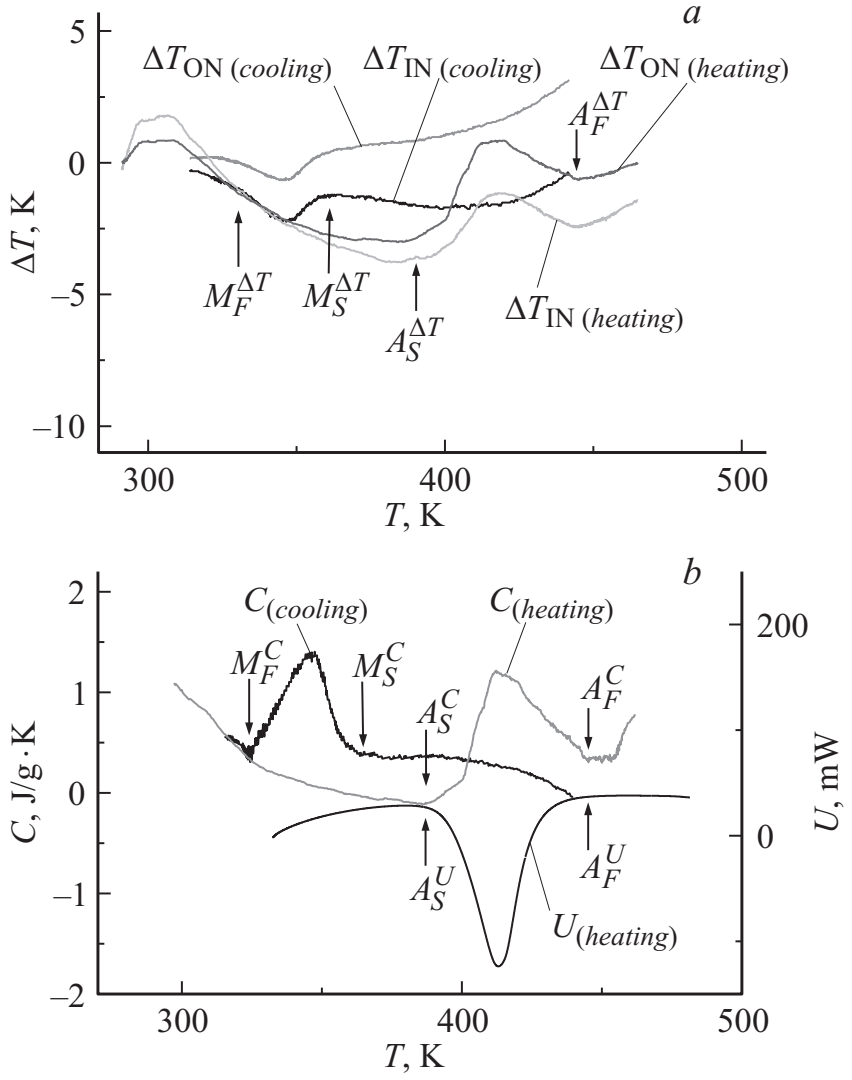

Рис. 2. Зависимости $\Delta T(T)$ при изменении температуры без нагрузки, определенные по внутренним (ON) и по внешним (IN) вставкам $(a)$; зависимости $C(T)$ и $U(T)$ при изменении температуры без нагрузки $(b)$.

можно утверждать, что при охлаждении имеет место превращение $B 2 \rightarrow B 19^{\prime}$, а при нагреве $B 19^{\prime} \rightarrow B 2$, где $B 2$ - высокотемпературная кубическая фаза типа $\mathrm{CsCl}$, а $B 19^{\prime}$ - низкотемпературная моноклинная фаза. Кроме того, весь образец протестировали (после такой же закалки) на аппарате C80 Calvet Calorimeter c разрешением $0.1 \mu \mathrm{W}$, при нагреве с темпом $1 \mathrm{grad} / \mathrm{min}$. Ограничились только этапом нагрева в том же диапазоне температур, что и в предыдущем случае. Результаты представлены на рис. $2, b$ зависимостью $U(T)$ теплового потока от температуры. Здесь обратное превращение фиксируется при температурах 380-445 K, его величина составила $q^{H}=30.5 \mathrm{~J} / \mathrm{g}$. Сравнение с данными тестирования тонкой пластинки показывает, что величины $q^{H}$ в обоих случаях практически одинаковые. Также практически совпадают и температуры начала теплопоглощения при нагреве. Однако температуры окончания этого процесса разнятся. Очевидно, это следует связывать с размерами и массой измеряемого объекта, так как массивный образец обладает большей тепловой инерцией.

Затем исследовали теплофизические свойства образца по описанной методике. Сначала изучили поведение материала без нагрузки. Температуры фиксировали с ин- 
тервалом времени $\Delta t=10 \mathrm{~s}$. На электронагревательном приборе устанавливали такое электрическое напряжение $E$, что нагревание во время протекания обратного фазового перехода осуществлялось с темпом приблизительно $2 \mathrm{grad} / \mathrm{min}$. Результаты даны на рис. 2, $a$ в виде зависимостей $\Delta T_{\mathrm{ON}}(T)$ и $\Delta T_{\mathrm{IN}}(T)$. Видно, что в целом при нагревании и охлаждении внешний и внутренний контуры позволяют получать сходные результаты, при этом выделяются аномальные участки, указывающие на протекание экзотермического (при нагревании) и эндотермического (при охлаждении) процессов с характеристическими температурами: для обратной реакции $A_{S}^{\Delta T}=386 \mathrm{~K}, A_{F}^{\Delta T}=444.5 \mathrm{~K}$, для прямой реакции $M_{S}^{\Delta T}=363 \mathrm{~K}, M_{F}^{\Delta T}=324.5 \mathrm{~K}$. Сравнение с результатами тестирования показывает, что эти температуры несколько отличаются от температур, найденных на малых образцах в калориметре DSC 822 $\mathrm{e}$ METTLER TOLEDO. Однако их отличие от температур, полученных при тестировании целого образца в установке C80 Calvet Calorimeter, мало́ и не превышает $6 \mathrm{~K}$ для величины $A_{S}$. Очевидно, здесь сказывается относительно высокая тепловая инерционность используемой конструкции (рис. 1). Теплоемкость $C$ никелида титана определяли по формуле (11), при этом использовали данные для удельной теплоемкости $C_{P}$ для меди и стали при разных температурах из справочника [8]. Зависимости $C(T)$ представлены на рис. 2, $b$. Видно хорошее соответствие расположения на температурной оси аномалий на графиках $U(T)$ и $C(T)$. Определяя величину $q$ как площадь под пиками на кривых $C(T)-$ в интервале температур $M_{S}^{C}-M_{F}^{C}$ для прямой реакции и в интервале температур $A_{S}^{C}-A_{F}^{C}$ для обратной реакции, мы получили $q^{H}=49 \pm 2 \mathrm{~J} / \mathrm{g}$ и $q^{C}=38 \pm 4 \mathrm{~J} / \mathrm{g}$, что в среднем соответственно в 1.53 и в 1.26 раза больше, чем было определено в тестовых калориметрических опытах. Видимо, это различие обусловлено несовершенством используемой методики. Однако, величины $q^{H}$ и $q^{C}$ качественно согласуются с калориметрическими данными. Поэтому можно сказать, что представленная методика, хотя не позволяет получить точные значения теплоты перехода, но дает представление об их уровне, а значит при сходных условиях позволяет их сравнивать.

Исходя из этого были проведены дальнейшие испытания по схеме: нагрев до температуры приблизительно $465 \mathrm{~K}$ без нагрузки, нагружение до величины напряжения кручения $\tau$, охлаждение при $\tau=$ const до комнатной температуры, разгрузка. При этом измеряли величину сдвига $\gamma$, происходящего в процессе прямого мартенситного превращения. Во время последующего нагревания образца в ненагруженном состоянии вследствие обратного мартенситного превращения происходил деформационный возврат. Напряжение $\tau$ от опыта к опыту увеличивали по программе 0, 35, 53, 70, 88, $103 \mathrm{MPa,}$ а потом уменьшали в обратной последовательности. После первой серии опытов образец заново отожгли и закалили по прежнему режиму и повторили эксперименты, которые дали такие же результаты. На рис. 3 пред-

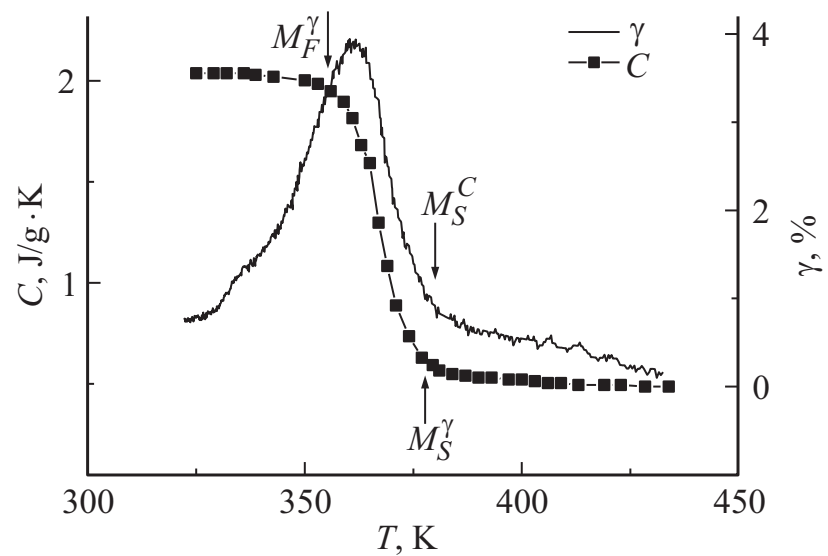

Рис. 3. Зависимости $C(T)$ и $\gamma(T)$ при охлаждении под постоянным напряжением $35 \mathrm{MPa}$.

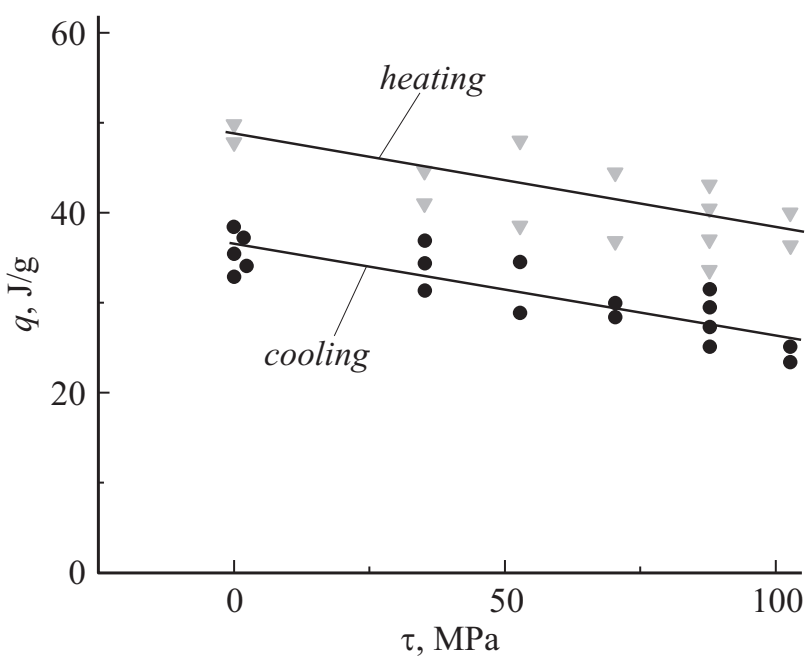

Рис. 4. Зависимости величины $q^{C}$ от действующего напряжения $\tau$ и величины $q^{H}$ от напряжения $\tau$, которое действовало во время предварительного охлаждения.

ставлены характерные графики зависимости $C$ и $\gamma$ от температуры $T$ для случая охлаждения при $\tau=35 \mathrm{MPa}$. Здесь видно, что в целом тепловые аномалии совпадают с деформационными эффектами на температурной шкале и четко выявляются. При этом температура начала накопления деформации $M_{S}^{\gamma}$ практически совпадает с температурой $M_{S}^{C}$ начала аномалии на кривой $C(T)$.

На основе полученных графиков $C(T)$ по описанной выше методике были вычислены значения $q$ для всех проведенных опытов. Результаты расчетов представлены на рис. 4 в виде зависимости величин $q^{C}$ и $q^{H}$ от напряжения $\tau$. Видно, что с увеличением нагрузки от 0 до $103 \mathrm{MPa} \mathrm{величины} q^{C}$ и $q^{H}$ практически одинаково уменьшаются в среднем в 1.3 раза: $q^{C}$ изменяется с 37 до $26 \mathrm{~J} / \mathrm{g}$, а $q^{H}$ - с 49 до $40 \mathrm{~J} / \mathrm{g}$, причем характер этого уменьшения - линейный. Отметим, что аналогичное по величине падение теплоты обратного фазового перехода 
$B 19^{\prime} \rightarrow B 2$ в никелиде титана, предварительно деформированного и помещенного в ситуацию защемления, было зафиксировано и в работе [4].

\section{Обсуждение полученных результатов}

Уменьшение энтальпии перехода $q^{C}$ под нагрузкой не может быть связано с возникновением в материале дефектов, в результате которого объем, охваченный превращением, уменьшается, поскольку напряжения не подавляли способность материала к накоплению фазовой деформации в процессе мартенситных превращений. Об этом свидетельствуют данные на рис. 5, где представлены зависимости величины деформации кручения $\gamma$ при нагреве и охлаждении. Здесь линия 1 относится к экспериментам, в которых напряжение $\tau$ от опыта к опыту увеличивали, а линия 2 - когда напряжение от опыта к опыту уменьшали. Видно, что во всех случаях величина деформационного возврата при нагреве практически равна деформации, накопленной при охлаждении, а в ряде случаев первая даже несколько превышает вторую. Это значит, что приложение напряжений не приводило к повреждению материала даже при максимальных в наших опытах значениях $\tau=103 \mathrm{MPa}$, и он всегда сохранял полную способность к фазовым превращениям.

Рассматриваемый эффект не может быть связан и с упорядочиванием поликристалла во время охлаждения под нагрузкой. Вообще говоря, выстраивание кристаллов во время прямого превращения в определенном ориентационном направлении, задаваемом напряжением, приводит к тому, что при прочих равных условиях энтропия образца в низкотемпературном (мартенситном) состоянии, полученном при охлаждении под напряжением, будет меньше, чем в случае, когда охлаждение осуществлялось без нагрузки: $S_{M}(\tau>0)<S_{M}(\tau=0)$. Поэтому скачок энтропии (а значит и энтальпия перехода) при наличии напряжений будет больше, чем без них.

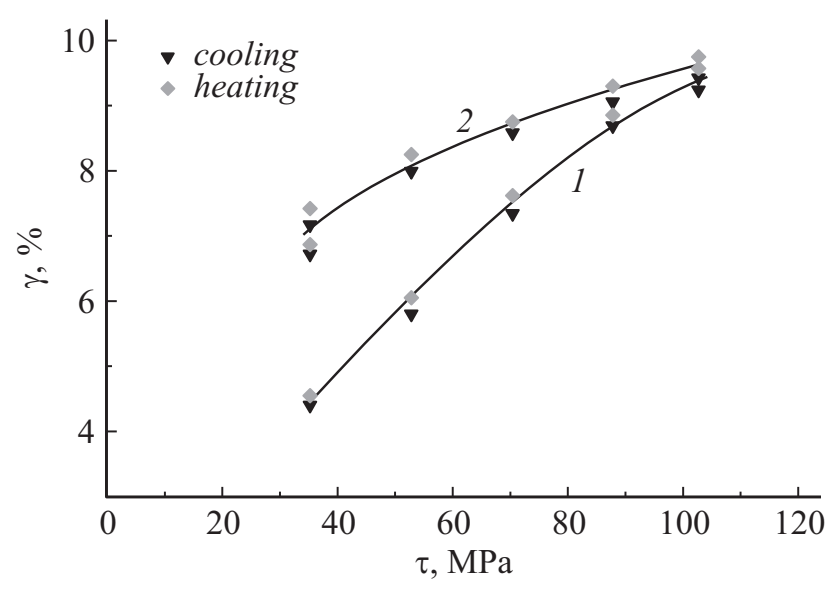

Рис. 5. Зависимости $\gamma(\tau)$, когда напряжение $\tau$ от опыта к опыту увеличивали (линия 1), и когда его уменьшали (линия 2).
Далее следует отвести предположение, сделанное в работе [4], о том, что теплота перехода может изменяться вследствие упругого искажения формы, поскольку, как об этом уже говорилось во введении, такой подход предполагает скачкообразный характер зависимости $q(\tau)$. Опыт же дает здесь плавную и практически линейную зависимость (рис. 4). При этом аналогичные результаты для случая растяжения монокристалла из $\mathrm{CuAlNi}$ были получены и в [3].

Наконец, в работе [5] было доказано, что смещение напряжениями температуры фазового равновесия (согласно правилу Клаузиуса-Клапейрона) тоже не может быть основанием для объяснения рассматриваемого явления.

В то же время следует отметить, что если для монокристалла $\mathrm{CuAlNi}$ при растяжении имеет место возрастающая зависимость $q(\tau)$ [3], то при изгибе она убывающая [5]. Для поликристалла TiNi она убывающая и при изгибе [4] и при кручении, как это следует из настоящих исследований. Поэтому, судя по всему, важным оказывается некоторый синтез как схемы напряженного состояния, так и структурных особенностей материала. В результате такого синтеза возникает пока неясная особенность возникновения и взаимодействия межзеренных и межфазных границ, которая может стать определяющей в зависимости $q(\tau)$. Об этом свидетельствует и то: что в наших опытах темп уменьшения величин $q^{C}$ и $q^{H}$ с ростом напряжения $\tau$ одинаковый (рис. 4). Действительно, поскольку прямое превращение в описанных экспериментах осуществлялось под напряжением, а обратное - в свободном состоянии, причину уменьшения величин $q^{C}$ и $q^{H}$ следует искать в особенностях строения мартенсита, полученного в условиях действия напряжения, а не в величине работы, производимой внешней нагрузкой.

В целом следует признать, что это явление пока не имеет полного и ясного обоснования и требуются дополнительные исследования по его изучению.

\section{Заключение}

1. Создана усовершенствованная версия дифференциального термического анализа, позволяющая изучать влияние напряжений кручения на теплоту фазовых превращений в твердых телах в интервале температур от комнатной до приблизительно $500 \mathrm{~K}$.

2. В изученном сплаве никелида титана увеличение напряжений кручения от 0 до $103 \mathrm{MPa} \mathrm{приводит} \mathrm{к} \mathrm{линей-}$ ному уменьшению энтальпии прямого мартенситного превращения приблизительно в 1.3 раза. В такой же степени уменьшается и энтальпия обратного превращения при последующем нагреве без нагрузки.

Авторы благодарят Н.Н. Реснину и ресурсный центр СПбГУ „Термогравиметрические и калориметрические методы исследований“ за помощь в калориметрических испытаниях. 


\section{Список литературы}

[1] Ortin J. // Thermochim. Acta. 1987. Vol. 121. P. 397.

[2] Ortin J., Planes A. // Acta Metallurg. 1989. Vol. 37. N 5. P. 1433.

[3] Ortin J. et al. // Phyl. Mag. A. 1992. Vol. 65. P. 461.

[4] Егоров С.А. // ЖТФ. 2006. Т. 76. Вып. 8. С. 37.

[5] Николаев В.И., Егоров В.И., Малыгин Г.А., Пульнев С.А. // ФТТ. 2010. Т. 52. Вып. 11. С. 2263.

[6] Friend C.M., Manosa Li, Ortin J., Planes A. // J. Phys. IV. 1991. Vol. 1. P. 4-71.

[7] Хачиу В.Р., Пушин В.Г., Кондратьев В.В. Никелид титана: структура и свойства. М.: Наука, 1992. 160 с.

[8] Свойства металлов при высоких температурах / Под ред. В.Е. Зиновьева. Справ. изд., М.: Металлургия, 1989. 384 с. 